\title{
IMIIII The Effectiveness of a Physician-Only and Physician-Patient Intervention on Colorectal Cancer Screening Discussions Between Providers and African American and Latino Patients
}

\author{
Nancy C. Dolan, $\mathrm{MD}^{7}$, Vanessa Ramirez-Zohfeld, $\mathrm{MPH}^{7}$, Alfred W. Rademaker, PhD ${ }^{2,3}$, \\ M. Rosario Ferreira, $M D^{4}$, William L. Galanter, $M D, P h D^{5}$, Jonathan Radosta, $M D^{5}$, \\ Milton "Mickey" Eder, PhD', and Kenzie A. Cameron, $P h D, M P H^{1,2,3,7}$ \\ 'Division of General Internal Medicine and Geriatrics, Department of Medicine, Northwestern University Feinberg School of Medicine, Chicago, IL, \\ USA; ${ }^{2}$ Department of Preventive Medicine, Northwestern University Feinberg School of Medicine, Chicago, IL, USA; ${ }^{3}$ Robert H. Lurie Comprehensive \\ Cancer Center, Northwestern University, Evanston, IL, USA; ${ }^{4}$ Division of Gastroenterology, Northwestern University Feinberg School of Medicine, \\ Chicago, IL, USA; ${ }^{5}$ Division of General Internal Medicine, Department of Medicine, University of Illinois Hospital \& Health Sciences System, Chicago, \\ IL, USA; ${ }^{6}$ Department of Family Medicine and Community Health, University of Minnesota, Minneapolis, MN, USA; ${ }^{7}$ Department of Medical Social \\ Sciences, Northwestern University Feinberg School of Medicine, Chicago, IL, USA.
}

BACKGROUND: Physician recommendation of colorectal cancer (CRC) screening is a critical facilitator of screening completion. Providing patients a choice of screening options may increase CRC screening completion, particularly among racial and ethnic minorities.

OBJECTIVE: Our purpose was to assess the effectiveness of physician-only and physician-patient interventions on increasing rates of CRC screening discussions as compared to usual care.

DESIGN: This study was quasi-experimental. Clinics were allocated to intervention or usual care; patients in intervention clinics were randomized to receipt of patient intervention.

PARTICIPANTS: Patients aged 50 to 75 years, due for CRC screening, receiving care at either a federally qualified health care center or an academic health center participated in the study.

INTERVENTION: Intervention physicians received continuous quality improvement and communication skills training. Intervention patients watched an educational video immediately before their appointment.

MAIN MEASURES: Rates of patient-reported 1) CRC screening discussions, and 2) discussions of more than one screening test.

KEY RESULTS: The physician-patient intervention $(n=167)$ resulted in higher rates of CRC screening discussions compared to both physician-only intervention ( $n=183 ; 61.1 \%$ vs.50.3\%, $p=0.008$ ) and usual care ( $n=153 ; 61.1 \%$ vs. $34.0 \% p=0.03$ ). More discussions of specific CRC screening tests and discussions of more than one test occurred in the intervention arms than in usual care (44.6\% vs. $22.9 \%, p=0.03)$ and (5.1\% vs. $2.0 \%$, $p=0.036)$, respectively, but discussion of more than one test was uncommon. Across all arms, 143 patients (28.4\%) reported discussion of colonoscopy only; 21 $(4.2 \%)$ reported discussion of both colonoscopy and stool tests.

Received October 21, 2014

Revised March 16, 2015

Accepted April 15, 2015

Published online May 19, 2015
CONCLUSIONS: Compared to usual care and a physician-only intervention, a physician-patient intervention increased rates of CRC screening discussions, yet discussions overwhelmingly focused solely on colonoscopy. In underserved patient populations where access to colonoscopy may be limited, interventions encouraging discussions of both stool tests and colonoscopy may be needed.

KEY WORDS: colorectal cancer screening; health literacy; randomized trial; physician communication of preventive care.

J Gen Intern Med 30(12):1780-7

DOI: $10.1007 / \mathrm{s} 11606-015-3381-8$

(c) Society of General Internal Medicine 2015

\section{INTRODUCTION}

Colorectal cancer (CRC) is the second most common cause of cancer-related death in the United States. ${ }^{1}$ Effective screening tests include fecal occult blood tests (FOBT), flexible sigmoidoscopy, colonoscopy, or a combination of these tests, ${ }^{2-7}$ but remain underused, particularly among minorities and those with low income, no insurance, or no usual source of care. ${ }^{8}$ The U.S. Preventive Services Task Force currently recommends screening for $\mathrm{CRC}$ beginning at age 50 and continuing until age 75 , but does not endorse a specific test. ${ }^{9}$

A physician's recommendation of CRC screening tests is a major facilitator of CRC screening. ${ }^{10-12}$ More comprehensive physician CRC discussions have been identified as being associated with higher screening rates among adults aged 51 to 80 years who were overdue for screening. ${ }^{13}$ Discussions of appropriate screening options, however, are often inadequate. ${ }^{14-16}$ Furthermore, when these discussions are held, primary care physicians routinely recommend colonoscopy as their preferred test and rarely mention other tests. ${ }^{17-19}$ Recent research, however, demonstrates that providing patients a choice of screening options may increase CRC screening completion, particularly among racial and ethnic minorities. $^{20}$ 
We conducted a quasi-experimental trial to evaluate and compare the effectiveness of a physician-only and a physician-patient intervention on physicians' recommendations for CRC screening and actual rates of screening. This paper focuses on patients' self-report of physician-patient CRC screening discussions during a non-acute general internal medicine visit. We hypothesized that: patients receiving a physician-only or physician-patient intervention would be more likely to report any discussion of CRC screening with their physician and more likely to report discussion of more than one CRC screening strategy as compared to those in usual care. Furthermore, we hypothesized that patients receiving the physician-patient intervention would be more likely to report any discussion of CRC screening and more likely to report discussion of more than one CRC screening option, compared to those receiving the physician-only intervention.

\section{METHODS}

\section{Trial Design}

Clinics initially were cluster randomized to either physician intervention or usual care. Originally six clinics were involved, three serving primarily Hispanic/Latino $(\mathrm{H} / \mathrm{L})$ communities, and three serving primarily African American (AA) communities. We created three separate envelopes for the $\mathrm{H} / \mathrm{L}$ clinics and three for the AA clinics; participating clinics selected a prepared sealed envelope that identified them as either a usual care or an intervention clinic. We randomized two H/L and two AA clinics to intervention and one $\mathrm{H} / \mathrm{L}$ and one $\mathrm{AA}$ clinic to usual care. Patients at intervention clinics were further randomized to receipt of an educational video shown prior to the appointment, or no video. We used block randomization in blocks of eight for randomization to the patient intervention; a custom-designed applet on the interviewer's study laptop computer indicated to which arm an individual patient was to be randomized. The Institutional Review Boards at the Northwestern University Feinberg School of Medicine and the University of Illinois Hospital \& Health Sciences System approved this study.

\section{Study Setting and Participants}

The initial study sites were six community based health centers within Access Community Health Network (ACCESS). ACCESS is a Federally Qualified Health Center (FQHC) with a network of approximately 50 clinics serving the underserved in the greater Chicago area. Clinic sites were selected to minimize any possibility of patient crossover and to include three ACCESS clinics serving primarily $\mathrm{H} / \mathrm{L}$ patients and three serving primarily AA patients. Due to clinic closures amidst reorganization within the multi-site Network, within the first year of the study, three of the original clinics dropped out. These sites were replaced with clinics chosen based on similarity of patient population between the original randomized clinics (one usual care and two intervention sites) and the nonrandomized replacement clinics (which consisted of one usual care and one intervention site). Further, in order to increase recruitment, an academic health center (staffed by both attending and resident physicians) was added and served as both a usual care and an intervention site. The decision to use the academic health center as both a usual care and an intervention site was due to differences in the patient populations of the FQHC and academic sites. A total of 15 attending physicians practiced at the academic health center. Three of those were deemed ineligible, as two were affiliated with the trial and one was leaving the practice. Because of the need for a larger sample size in the intervention arms, the one attending who had the greatest number of patients was allocated to the physician intervention. The remaining 11 attending physicians were randomized to usual care or intervention via use of a random number generator. Residents were identified as being usual care or intervention, based on their supervising attending.

Physician participants were Internal Medicine or Family Practice physicians practicing at the intervention clinics. The $\mathrm{FQHC}$ clinics had from one to five physicians with a mean of two physicians per clinic; a total of 51 resident physicians (32 usual care, 19 intervention) participated at the academic health center.

Patient participants were English-speaking or Spanishspeaking patients between the ages of 50 and 75 years, who were registered for a non-acute physician appointment at a participating clinic, and were not up to date with CRC screening. In addition, patients were required to be established patients, defined as having been seen at the clinic at least twice in the previous 2 years. Patients were excluded if they were unable to view study materials (e.g., blind, deaf, too ill); had a personal or family history of CRC, had polyps or inflammatory bowel disease; were deemed ineligible for screening by physician; or saw a non-study physician. Exclusion based on up-to-date CRC screening and personal or family history was based both on patient self-report and chart review.

\section{Interventions}

The physician intervention, a version of which was previously pilot tested in both a Veterans Affairs (VA) internal medicine practice and an FQHC population, ${ }^{21-23}$ consisted of communication skills training and continuous quality improvement (CQI). The communication skills training was designed to help improve physician communication about CRC and CRC screening among ethnically diverse patients with inadequate or limited health literacy. The accompanying CQI component consisted of medical record audit and feedback on CRC screening recommendations and screening test completion. Physicians in intervention clinics attended an initial onehour session, and then between two to four additional "booster" sessions every 5 to 7 months. A detailed outline of these sessions is available as an appendix. 
The initial one-hour training session included a review of the study, current CRC screening guidelines, and communication strategies for patients with low or limited health literacy. Examples of communication strategies included the teachback method, using simplified language, and employing visual aids. ${ }^{24}$ During one of the booster sessions, research on the potential benefits of providing a choice of screening tests options, especially in this population, was reviewed. During three of the one-hour booster sessions rates of CRC recommendation, type of test recommended, and rates of CRC screening completion, along with type of screening completed, were presented to each participating physician. The data were presented in a tabular form showing the clinic-level averages as well as the averages across all intervention clinics. Rates were presented in a way that physicians could see how they were doing over time.

Patients of intervention physicians who were randomized to the physician-patient intervention viewed a brief educational video prior to the appointment. The video included information on CRC, rationale for screening, screening test options, and incorporated plain language, graphic design, and audio voice-over to overcome literacy limitations. It encouraged patients to discuss screening options with their provider, noting that the important behavior was to be screened, regardless of type of test. The video was previously pilot tested among both English-speaking and Spanish-speaking patients with limited literacy, and was shown to improve patient knowledge and willingness to consider CRC screening (video available at: http://gim-geriatrics.medicine.northwestern.edu/resources/ get-screened-colorectal-cancer). ${ }^{22}$

\section{Recruitment Strategy}

The trial was conducted from September 2010 to January 2013. Research assistants reviewed daily appointment lists at the study clinics. Potentially eligible patients were approached and invited to complete a brief screening interview to verify eligibility for the study. After eligible patients signed informed consent, same-dayin-person interviews were conducted by the research assistants both immediately before and after the appointment. Patients were given the choice of completing the interview in Spanish or English. Patients randomized to watch the video were shown the video in the language they requested for the interview.

\section{Measures}

As part of the larger trial, the pre-visit structured interview included questions on socio-demographic information, a health literacy assessment, ${ }^{25}$ and items assessing knowledge, attitudes and self-efficacy regarding CRC and CRC screening. At the end of the pre-visit interview, patients were randomized to intervention and usual care arms. The post-visit structured interview included specific questions regarding discussion of CRC screening: "Did you and your doctor talk about getting tested for colorectal cancer today?", "Did you and your doctor talk about specific tests?" and "Which tests?" Participants responses to the first two items were dichotomized into "yes" and "no" responses; the third question was posed as an open-ended question and interviewers identified whether the participant noted stool tests, flexible sigmoidoscopy, colonoscopy, or some combination of those options. The pre-visit and post-visit interviews occurred immediately before and after the physician visit. Patients randomized to the video viewed it after the pre-visit interview and immediately before the visit. Physicians were not informed as to which of their patients were randomized to the educational video; however, we did not prevent patients from mentioning it to their physician at the time of their appointment.

\section{Data Analyses}

All analyses were performed using SAS 9.2 (SAS Institute, Inc., Cary, NC). The usual care arm was compared to any intervention (i.e., physician intervention, physician-patient intervention) using a repeated measures generalized linear model accounting for clustering by clinic. The two intervention arms were compared using a mixed generalized linear model with intervention as the fixed effect and clinic as the random effect. We adjusted for age when comparing the two intervention arms.

\section{RESULTS}

\section{Patient Recruitment and Follow-up}

A total of 73,497 patients were assessed for eligibility; 566 were allocated ( $n=168$ to usual care, $n=398$ to intervention), and 503 completed both the pre-tests and post-tests, had eligibility verified, and were included in the analysis (Fig. 1). Reasons for exclusion included not meeting inclusion criteria, not showing up for the appointment, coming in for an appointment at a time when no study research assistants were available to recruit participants, and declining participation. The most common reasons for not meeting inclusion criteria included age $(n=58,057)$, being scheduled with a non-study physician $(n=3,815)$, being up-to-date on screening $(n=3,335)$, and being new to the clinic $(n=1,119)$.

\section{Participant Characteristics}

The mean age of patients completing both pre-test and postvisit surveys was 57.8 years $(\mathrm{SD}=0.28) ; 74.0 \%$ were female, $54.2 \%$ were Non-Hispanic Black, $45.8 \%$ were Hispanic/ Latino, $66.8 \%$ had household incomes of less than $\$ 20,000$, $39.0 \%$ had inadequate health literacy, and $30.4 \%$ had no insurance. Patient characteristics were similar across study arms with respect to age, sex, race, years of education, household income, health status, health literacy and type of health care system. Patients receiving the physician-patient intervention were younger than those receiving physician-only intervention (Table 1). 


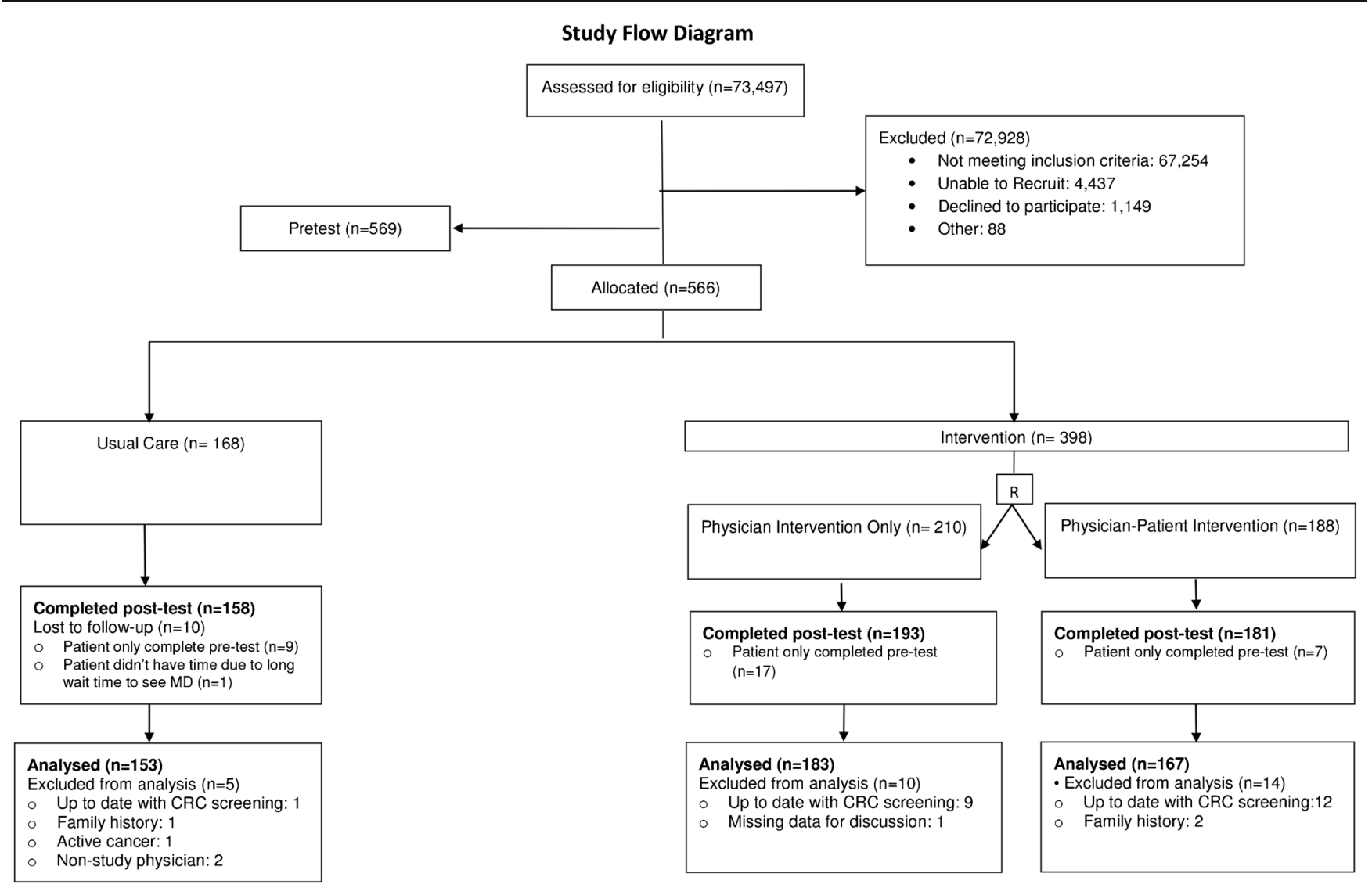

Figure 1. Participant recruitment and flow chart.

\section{CRC Screening Discussion Rates (Table 2)}

Reported rates of CRC discussions were higher in the physician-patient intervention arm compared to the physician-only intervention $(61.1 \%$ vs. $50.3 \%$, $p=0.008)$ or usual care $(61.1 \%$ vs. $34.0 \%, p=0.03)$ arms. Of note, data were also analyzed only for the community FQHCs, excluding the academic site to identify whether or not the results held when the academic site was excluded. Results were similar, and remained statistically significant when comparing the interventions both with and without the video $(p=0.049)$. There were no significant differences in patient reported discussion of specific tests or more than one screening test between the physician-patient intervention and the physician-only intervention. However, patients of intervention physicians, regardless of whether or not they received the patient intervention, were more likely to report discussing a specific CRC test with their physician than those in the usual care arm (44.6\% vs. $22.9 \%, p=0.03$ ).

Across all arms, colonoscopy only was the most common test discussed; 143 patients (28.4\%) reported discussion of colonoscopy only; 21 (4.2\%) reported discussion of both colonoscopy and stool tests. Because only one patient reported discussing flexible sigmoidoscopy with the physician, it was analyzed with colonoscopy.
Although a significant difference was observed, as hypothesized, between patients in the intervention arms or usual care ( $5.1 \%$ and $2.0 \%$ respectively, $p=0.036$ ), rates of discussion of more than one screening test remained low. Contrary to our initial hypothesis, the more intensive physician-patient intervention was not associated with increased discussion of more than one screening test compared to the physician-only intervention $(5.4 \%$ to $4.9 \%, p=0.99)$.

\section{DISCUSSION}

The patient-physician CRC screening intervention, which included communication skills training and CQI for physicians, and a pre-visit educational video for patients, resulted in higher self-reported rates of CRC screening discussions compared to either a physician-only intervention or usual care. Patients of intervention physicians, regardless of whether they received the patient intervention, were more likely than usual care to report discussing a specific CRC test and more than one screening test with their physician. Discussions of tests other than colonoscopy, however, were low across all arms.

The majority of interventions designed to increase CRC screening discussions, recommendations, and ultimately screening test completion, have targeted either physicians or patients. Interventions targeting physicians have included academic detailing, ${ }^{26,27}$ chart audit and feedback, ${ }^{28,29}$ reminder 
Table 1. Participant Demographics by Randomization Group, Including Only Those Who Completed Post-Visit Survey*

\begin{tabular}{|c|c|c|c|c|c|c|}
\hline Characteristic & Usual care & Intervention & p value & $\begin{array}{l}\text { Physician } \\
\text { intervention only }\end{array}$ & $\begin{array}{l}\text { Physician-patient } \\
\text { intervention }\end{array}$ & p value \\
\hline Sample size & 153 & 350 & & 183 & 167 & \\
\hline Age, mean (SE) & $57.80(0.75)$ & $57.80(0.53)$ & 0.99 & $58.58(1.08)$ & $56.95(1.09)$ & 0.01 \\
\hline Sex, n $(\%)$ & 153 & 350 & & 183 & 167 & \\
\hline Female & $117(76.5)$ & $255(72.9)$ & 0.82 & $130(71.0)$ & $125(74.9)$ & 0.26 \\
\hline Race/ethnicity, n (\%) & 141 & 342 & 0.99 & 181 & 161 & 0.68 \\
\hline Hispanic/Latino & $64(45.4)$ & $157(45.9)$ & & $85(47.0)$ & $72(44.7)$ & \\
\hline Non-hispanic black & $77(54.6)$ & $185(54.1)$ & & $96(53.0)$ & $89(55.3)$ & \\
\hline Survey language, n (\%) & 153 & 350 & 0.73 & 183 & 167 & 0.43 \\
\hline Spanish & $60(39.2)$ & $146(41.7)$ & & $80(43.7)$ & $66(39.5)$ & \\
\hline Years of education, n (\%) & 151 & 348 & 0.70 & 182 & 166 & 0.79 \\
\hline $0-6$ years & $41(27.2)$ & $67(19.3)$ & & $33(18.1)$ & $34(20.5)$ & \\
\hline $7-12$ years & $40(26.5)$ & $112(32.2)$ & & $61(33.5)$ & $51(30.7)$ & \\
\hline $13+$ years & $70(46.4)$ & $169(48.6)$ & & $88(48.4)$ & $81(48.8)$ & \\
\hline Household income, n (\%) & 150 & 341 & 0.94 & 179 & 162 & 0.35 \\
\hline$<\$ 20,000$ & $96(64.0)$ & $232(68.0)$ & & $125(69.8)$ & $107(66.1)$ & \\
\hline$\$ 20,000-40,000$ & $22(14.7)$ & $43(12.6)$ & & $23(12.9)$ & $20(12.3)$ & \\
\hline$>\$ 40,001$ & $13(8.7)$ & $14(4.1)$ & & $6(3.3)$ & $8(4.9)$ & \\
\hline Unknown & $19(12.6)$ & $52(15.3)$ & & $25(14.0)$ & $27(16.7)$ & \\
\hline Health status, n (\%) & 152 & 349 & 0.97 & 183 & 166 & 0.36 \\
\hline Poor & $23(15.1)$ & $47(13.5)$ & & $23(12.6)$ & $24(14.5)$ & \\
\hline Fair & $73(48.0)$ & $182(52.2)$ & & $98(53.6)$ & $84(50.6)$ & \\
\hline Good & $38(25.0)$ & $85(24.4)$ & & $42(23.0)$ & $43(25.9)$ & \\
\hline Very good & $16(10.5)$ & $22(6.3)$ & & $10(5.5)$ & $12(7.2)$ & \\
\hline Excellent & $2(1.3)$ & $13(3.7)$ & & $10(5.5)$ & $3(1.8)$ & \\
\hline Health literacy, n (\%) & 153 & 350 & & 183 & 167 & 0.29 \\
\hline Inadequate & $50(32.7)$ & $146(41.7)$ & 0.76 & $81(44.3)$ & $65(38.9)$ & \\
\hline Marginal/adequate & $91(59.5)$ & $171(48.9)$ & & $85(46.5)$ & $86(51.5)$ & \\
\hline Missing & $12(7.8)$ & $33(9.4)$ & & $17(9.3)$ & $16(9.6)$ & \\
\hline Health care system, n (\%) & 153 & 350 & 0.76 & 183 & 167 & 0.64 \\
\hline Community & $92(60.1)$ & $305(87.1)$ & & $158(86.3)$ & $147(88.0)$ & \\
\hline Academic & $61(39.9)$ & $45(12.9)$ & & $25(13.7)$ & $20(12.0)$ & \\
\hline Insurance, n (\%) & 153 & 350 & 0.52 & 183 & 167 & 0.63 \\
\hline Yes & $90(58.8)$ & $260(74.3)$ & & $134(73.2)$ & $126(75.5)$ & \\
\hline
\end{tabular}

${ }^{*} N$ varies slightly among demographic characteristics due to missing data

systems, ${ }^{30}$ and communication skills training. ${ }^{21,29}$ Pre-visit patient-level interventions in patients of lower socioeconomic status, which have been effective in increasing CRC screening discussions/recommendations, include activation counseling, ${ }^{31,32}$ computer-delivered tailored intervention ${ }^{33}$ and decision aids. ${ }^{34}$ Modest increases (7-27\%) in CRC discussions and/or recommendations with these interventions have been reported. ${ }^{31-34}$

Our current study is unique in targeting both physicians and patients; few prior studies have done so. ${ }^{21,35,36}$ Aragones et al. recently showed that a CRC educational video and brochure in Spanish and a patient-delivered physician reminder was associated with a trend toward an increase in physician CRC recommendation rate and a significant increase in CRC test completion. ${ }^{36}$ Ferreira et al. completed a combined intervention study similar to the current study, but in a single, academic, urban VA clinic. ${ }^{21}$ In that study, compared to usual care, patients of intervention physicians were more likely than those in usual care to have a recommendation for a screening test documented in the chart. Due to difficulty with implementation of the patient-directed component of the intervention, however, they were unable to assess the effectiveness of the combined intervention compared to the physician-only intervention. As we hypothesized, in the current study, the

Table 2 Participant Self-Report Of Discussion of CRC Screening by Arm

\begin{tabular}{|c|c|c|c|c|c|c|}
\hline & Usual care & Intervention & p value & $\begin{array}{l}\text { Physician } \\
\text { intervention only }\end{array}$ & $\begin{array}{l}\text { Physician-patient } \\
\text { intervention }\end{array}$ & p value \\
\hline Sample size & 153 & 350 & & 183 & 167 & \\
\hline \multicolumn{7}{|l|}{ Discussion*, n (\%) } \\
\hline Yes & $52(34.0 \%)$ & $194(55.4 \%)$ & 0.07 & $92(50.3 \%)$ & $102(61.1 \%)$ & 0.008 \\
\hline No & $101(66.0 \%)$ & $156(44.6 \%)$ & & $91(49.7 \%)$ & $65(38.9 \%)$ & \\
\hline Specific test discussed, n (\%) & 153 & 350 & $0.03^{*}$ & 183 & 167 & $0.16^{\ddagger}$ \\
\hline No discussion/no test specified ${ }^{\dagger}$ & $118(77.1 \%)$ & $194(55.4 \%)$ & & $109(59.7 \%)$ & $85(50.9 \%)$ & \\
\hline Stool test only & $0(0 \%)$ & $27(7.7 \%)$ & & $9(4.9 \%)$ & $18(10.8 \%)$ & \\
\hline Colonoscopy only & $32(20.9 \%)$ & $111(31.7 \%)$ & & $56(30.6 \%)$ & $55(32.9 \%)$ & \\
\hline Stool test and colonoscopy & $3(2.0 \%)$ & $18(5.1 \%)$ & & $9(4.9 \%)$ & $9(5.4 \%)$ & \\
\hline
\end{tabular}

${ }^{*} p=0.19$ for Usual Care vs. Physician intervention; $p=0.026$ for Usual Care vs. Physician-patient intervention

17 participants in Usual Care and 38 in Intervention reported a discussion, but reported that no CRC screening test was specified ${ }^{t} p=0.036$ comparing $5.1 \%$ to $2.0 \%$; $p=0.99$ comparing $5.4 \%$ to $4.9 \%$ 
combined physician-patient approach increased screening discussions compared to the physician only-intervention. These findings suggest that the pre-visit CRC screening video either activated patients to initiate physician-patient CRC screening discussions and/or improved their recall of these discussions.

The physician and patient components of the intervention were designed not only to increase CRC discussion and recommendation rates, but also to improve the quality of the discussions and increase discussion of more than one testing option. Research consistently demonstrates that a physician recommendation is a strong predictor of CRC screening completion, ${ }^{10-12}$ yet merely giving an order for CRC screening tests may not be enough, especially for patients with limited literacy skills. ${ }^{14}$ CRC screening discussions are often brief, omit important information, and do not include options other than colonoscopy. ${ }^{16,17,37,38}$ Recent research, however, shows that more comprehensive discussions of CRC screening by PCPs and providing patients with a choice of screening options may actually improve screening rates. ${ }^{13,20}$ To encourage physicians to discuss CRC screening test options with patients, the physician intervention emphasized the benefits of this practice and reviewed communication strategies for recommending and discussing each test. To educate patients on the test options, the pre-visit video provided patients with information on CRC screening test options and encouraged them to discuss these options with their provider, with emphasis placed on getting screened, not on a specific test.

As hypothesized, we found that patients of intervention physicians were more likely than usual care to report discussion of more than one screening test regardless of whether or not they saw the video, but surprisingly, the effect was very small. Similar to other settings, the test most commonly discussed was colonoscopy. Contrary to our hypothesis, the physician-patient intervention was no more effective than the physician-only intervention at increasing discussion of more than one test. Potential reasons are that patients perceived that their physicians preferred colonoscopy as the screening option and thus were reticent to initiate a discussion about FOBT if their physician did not bring it up, or that after learning about the options, patients themselves preferred colonoscopy.

There are many compelling reasons for recommending colonoscopy as a first-line test over FOBT: it has better test characteristics, it has to be performed only every 10 years compared with every year, and it is recommended as a firstline test by several professional societies. ${ }^{39,40}$ Colonoscopy, however, is costly and not always readily available. Furthermore, some research has proposed that many patients, especially those with low health literacy, prefer stool tests for screening. ${ }^{41,42}$ Patients who prefer stool tests over colonoscopy tend to cite ease and convenience as the most common reasons for their preference. Other reasons include the cost, discomfort, time, invasiveness and inconvenience associated with colonoscopy. ${ }^{41}$ In addition, there is evidence that limiting provider $\mathrm{CRC}$ recommendation to colonoscopy can result in lower CRC completion rate compared with a strategy of providing a recommendation for FOBT or a choice between the two, especially among racial and ethnic minorities. ${ }^{20}$ Inadomi and colleagues evaluated adherence to three initial provider CRC screening recommendations in a racially and ethnically diverse urban public health care system: FOBT, colonoscopy, or choice between the two. Patients who were recommended FOBT or choice were almost twice as likely to complete screening ( $67 \%$ and $69 \%$ respectively) compared to those who were recommended only colonoscopy (38\%) $(p<0.001)$. Baker et al. conducted a randomized trial of a multifaceted intervention to improve adherence to FOBT in an ethnically diverse, low income population, and found that an intervention that used provider feedback, standing orders, and reminder systems achieved an adherence rate of $82.2 \%$ compared to $37.3 \%$ in usual care $(p<0.001) .{ }^{43}$ This study demonstrates that high rates of adherence to FOBT can be obtained in low-income, underserved populations.

Because the different options for CRC screening are variably available and acceptable to patients, providing patients, particularly those who remain unscreened after many interactions or those with more barriers to colonoscopy, with a choice of options including FOBT might ultimately improve screening rates. Given the high proportion of low-income, uninsured patients in this study population, it is particularly important that more accessible stool tests be offered instead of focusing solely on colonoscopy.

Strengths of the study include a diverse, non-White patient population, the unique combined physician-patient intervention, and use of multiple recruitment sites. Limitations include the use of patient self-report to determine whether or not patient-physician CRC discussions took place and what tests were discussed, as well as the focus on only one physician visit. Results may therefore underestimate physician recommendation rates, as recommendations may have been made without a reported discussion and/or may be made during a future appointment. Patient self-report of CRC screening discussions immediately following a non-acute primary care visit is, however, arguably a more relevant outcome than physiciandocumented recommendation. If a patient does not recall the discussion immediately after the visit, a meaningful recommendation is unlikely to have occurred. It is well established that a patient's recall of a physician's recommendation for CRC screening is a strong predictor of actual screening and that the lack of such a recommendation and/or counseling results in less screening. Using data from the 2000 National Health Interview Survey, for example, Wee et al. showed that over $90 \%$ of patients who did not undergo screening did not recall being counseled to do so by their physicians in the preceding year. Findings from a recent observational study in Kaiser Permanente show that the content of the discussion at the time of recommendation also matters, with more comprehensive discussions (determined by patient recall) being associated with increased rates of screening. Although we have no information on who initiated the discussion or on the quality of 
the discussion, whether or not a specific test or more than one test was discussed gives us some indication of quality. Lastly, we met with several challenges inherent in conducting research in the underserved community setting, such as unexpected clinic closures and low recruitment; both required the addition of new clinics midway through the study. These conditions, however, are more relevant to real world clinical practice and further increase the external validity of the study.

Interventions that both increase CRC screening discussions and discussions of more than one screening option may help improve CRC screening, particularly among urban, low-income, underserved populations. Although our combined physician-patient intervention improved CRC discussion rates compared to physician-only or usual care, it was not successful at improving discussion of CRC tests other than colonoscopy. Promoting greater knowledge of alternate screening options may be especially important in communities with limited access to health care and more barriers to colonoscopy. In these settings, achieving high rates of screening is unlikely to occur if physicians continue with the strategy of recommending only colonoscopy. Comprehensive physician-patient CRC discussions, which include risk and benefits of colonoscopy and FOBT, however, may not be feasible, given the rapidly expanding demands on PCP time. Ultimately, to improve $\mathrm{CRC}$ rates among underserved populations, in addition to targeting providers and patients at the point of care, there is a need for multifaceted interventions that incorporate office systems, utilize office staff, and employ community outreach. With movement toward team-based care, targeting non-MDs (medical assistants, health educators, patient navigators) may be an alternative strategy for facilitating comprehensive discussion without further burdening PCPs. Implementing office systems, such as standing orders for screening tests and automated patient reminders, may help minimize the need for time-intensive, comprehensive CRC discussions.

\section{Acknowledgements:}

Contributors: We would like to acknowledge the efforts of our Research Assistants (Sabrina Collum, Mercedes Nodal, and Francisco Acosta), as well as the physicians, staff and patients of ACCESS Community Health Network and the University of Illinois Health Sciences System who participated in this study.

Source of Funding: This research project was funded by the National Cancer Institute (RO1 CA140177, Kenzie A. Cameron, PI), Trial Registration: Clinicaltrials.gov Identifier: NCTO1103479.

Prior Presentations: 2013 Society of General Internal Medicine; 25 April 2013; Denver, Colorado

2013 Midwest Society of General Internal Medicine; $13 v$ September 2013; Chicago, Illinois

2013 International Conference on Communication in Healthcare; 30 September 2013; Montreal, Canada

Conflicts of Interest: Kenzie Cameron is a co-Investigator on a grant entitled Dissemination of a Simulation-Based Mastery Learning Curriculum for Central Venous Insertion at Veteran Affairs Hospitals (Jeffrey H. Barsuk, PI); has a contract to Northwestern University from MERCI [Medical Error Reduction and Certification, Inc.]; and has received honoraria from the $\mathrm{NIH}$ (NIH, study section review).
Alfred Rademaker has a grant pending from Siemens to assess mammogram results and has received honoraria from Georgetown University (External Advisory Board), the American Association for Cancer Research (Workshop honorarium) and the NIH (NIH, study section review).

All other authors declare that they do not have a conflict of interest.

Corresponding Author: Nancy C. Dolan, MD; Division of General Internal Medicine and Geriatrics, Department of Medicine, Northwestern University Feinberg School of Medicine, 675 N. St. Clair St. Suite 18-200, Chicago, IL 60611, USA (e-mail: ndolan@nmff.org).

\section{REFERENCES}

1. American Cancer Society. Cancer Facts and Figures -2015 . Available at : http://www.cancer.org/acs/groups/content/@editorial/documents/document/acspc-044552.pdf Accessed April 16, 2015.

2. Whitlock EP, Lin JS, Liles E, Beil TL, Fu R. Screening for colorectal cancer: a targeted, updated systemic review for the U.S. Preventive Services Task Force. Ann Intern Med. 2008;149:638-658.

3. Mandel JS, Bond JH, Church TR, et al. Reducing mortality from colorectal cancer by screening for fecal occult blood. Minnesota Colon Cancer Control Study. N Engl J Med. 1993;328(19):1365-1371.

4. Hardcastle JD, Chamberlain JO, Robinson MH, et al. Randomised controlled trial of faecal-occult-blood screening for colorectal cancer. Lancet. 1996;348(9040): 1472-1477.

5. Kronborg $\mathbf{O}$, Fenger $\mathbf{C}$, Olsen $\mathbf{J}$, Jorgensen $\mathbf{O D}$, Sondergaard $\mathbf{O}$. Randomised study of screening for colorectal cancer with faecal-occultblood test. Lancet. 1996;348(9040):1467-1471.

6. Holme $\emptyset$, Løberg $\mathbf{M}$, Kalager $\mathbf{M}$, et al. Effect of flexible sigmoidoscopy screening on colorectal cancer incidence and mortality: a randomized clinical trial. JAMA. 2014;312(6):606-15.

7. Hundt S, Haug U, Brenner H, Hundt S, Haug U, Brenner H. Comparative evaluation of immunochemical fecal occult blood tests for colorectal adenoma detection. Ann Intern Med. 2009;150(3):162.

8. CDC. Vital signs: colorectal cancer screening test use - United States, 2012. MMWR Morb Mortal Wkly Rep. 2013;62(44):881-888.

9. U.S. Preventive Services Task Force. Screening for colorectal cancer: U.S. Preventive Services Task Force recommendation statement. Ann Intern Med. 2008;149(9):627-637.

10. Klabunde CN, Vernon SW, Nadel MR, Breen N, Seeff LC, Brown ML. Barriers to colorectal cancer screening: a comparison of reports from primary care physicians and average-risk adults. Med Care. 2005;43(9):939-944.

11. Wee CC, McCarthy EP, Phillips RX. Factors associated with colon cancer screening: the role of patient factors and physician counseling. Prev Med. 2005;41:23-29.

12. Guessous I, Dash C, Lapin P, Doroshenk M, Smith RA, Klabunde CN. Colorectal cancer screening barriers and facilitator $\mathrm{s}$ in older persons. Prev Med. 2010;50:3-10.

13. Mosen DM, Feldstein AC, Perrin NA, et al. More comprehensive discussion of CRC screening associated with higher screening. Am J Manag Care. 2013;19(4):265-271.

14. Ling BS, Trauth JM, Fine MJ, Mor MK, Resnick A, Braddock CH, et al. Informed decision-making and colorectal cancer screening: is it occurring in primary care? Med Care. 2008;46:S23-9.

15. Wackerbath SB, Tarasenko YN, Joyce JM, Haist SA. Physician colorectal cancer screening recommendations: an examination based on informed decision making. Patient Educ Counsel. 2007;66:43-50.

16. Wolf MS, Baker DW, Makoul G. Physician-patient communication about colorectal cancer screening. J Gen Intern Med. 2007;11:1493-9

17. McQueen A, Barthoomew, Greisinger AJ, et al. Behind closed doors: physician-patient discussions about colorectal cancer screening. J Gen Intern Med. 2009;24(11): 1228-35.

18. Klabunde CN, Lanier D, Nadel MR, McLeod C, Yuan G, Vernon Sw. Colorectal cancer screening by primary care physicians: recommendations and practices, 2006-2007. Am J Prev Med. 2009;37:8-16.

19. Hawley ST, Volk RJ, Krishnamurthy P, Jibaja-Weiss M, Vernon SW, Kneuper S. Preference for colorectal cancer screening among racially/ ethnically diverse primary care patients. Med Care. 2008;46:S10-S16.

20. Inadomi JM, Vijan S, Janz NK, et al. Adherence to colorectal cancer screening. A randomized clinical trial of competing strategies. Arch Intern Med. 2012;172:575-582. 
21. Ferreira MR, Dolan NC, Fitzgibbon ML, et al. Health care providerdirected intervention to increase colorectal cancer screening among veterans: results of a randomized controlled trial. J Clin Oncol. 2005;23(7):1548-1554.

22. Khankari $\mathbf{K}$, Eder $\mathbf{M}$, Osborn $\mathbf{C Y}$, et al. Improving colorectal cancer screening among the medically underserved: a pilot study within a Federally Qualified Health Center. J Gen Intern Med. 2007;22(10): 1410-1414.

23. Makoul G, Cameron KA, Baker DW, Francis L, Scholtens D, Wolf MS. A multimedia patient education program on colorectal cancer screening increases knowledge and willingness to consider screening among Hispanic/Latino patients. Patient Educ Couns. 2009;76:220-226.

24. Davis TC, Williams M, Marin E, Parker RM, Glass J. Health literacy and cancer communication. CA Canc J Clin. 2002;52:134-149.

25. Baker DW, Williams MV, Parker RM, et al. Development of a brief test to measure functional health literacy. Patient Educ Couns. 1999;38:33-42.

26. Dignan M, Shelton B, Slone SA, Tolle C, Mohammad S, Schoenberg N, Pearce K, Van Meter E, Ely G. Academic effectiveness of a primary care practice intervention for increasing colorectal cancer screening in Appalachian Kentucky. Prev Med. 2014;58:70-4.

27. Lane DS, Messina CR, Cavanagh MF, Chen JJ. A provider intervention to improve colorectal cancer screening in county health centers. Med Care. 2008;46:S109-16.

28. Sabatino SA, Larence B, Elder R, et al. Effectivenss of interventions to increase screening for breast. Cervical and colorectal cancers. Nine updated systematic reviews for the guide to community preventive services. Am J Prev Med. 2012;43(1):97-118.

29. Price-Haywood EG, Harden-Barrios J, Cooper LA. Comparative effectiveness of audit-feedback versus additional physician communication training to improve cancer screening for patients with limited health literacy. J Gen Intern Med. 2014;29(8):1113-21.

30. Sequist TD, Zaslavsky AM, Marshall R, Fletcher RH, Ayanian JZ. Patient and physician reminders to promote colorectal cancer screening: a randomized controlled trial. Arch Intern Med. 2009;169(4):364-71.

31. Katz ML, Fisher JL, Fleming K, Paskett ED. Patient activation increases colorectal cancer screening rates: a randomized trial among low-income minority patients. Cancer Epidemiol Biomarkers Prev. 2011;21:45-52.

32. Le V, Syed S, Vega KJ, Sharma T, Madhoun MF, Srinvasan N, Houchen CW. Patient prompting of their physicians resulted in increased colon cancer screening referrals. World J Gastrointest Oncol. 2014;6:257-62.
33. Christy SM, Perkins SM, Tong Y, Krier C, et al. Promoting colorectal cancer screening discussion. A randomized controlled trial. Am J Prev Med. 2013;44:325-329.

34. Schroy PC, Emmons KM, Peters E, et al. Aid-assisted decision making and colorectal cancer screening: a randomized controlled trial. Am J Prev Med. 2012;43(6):573-83.

35. Walsh JM, Salsazar R, Terdiman JP, et al. Promoting use of colorectal cancer screening tests. Can we change physican behavior? J Gen Intern Med. 2005;20:1097-101.

36. Aragones A, Schwartz MD, Shah NR, Gany FM. A randomized controlled trial of a multilevel intervention to increase colorectal cancer screening among Latino immigrants in a primary care facility. J Gen Intern Med. 2010;25(6):564-567.

37. Zapka JG, Klabunde CN, Taplin S, Yuan G, Ransohoff D, Kobrin S. Screening colonoscopy in the US: attitudes and practices of primary care physicians. J Gen Intern Med. 2012;27(9):1150-8.

38. Zapka JM, Klabunde CN, Arora NK, Yuan G, Smith JL, Kobrin SC. Physicians' colorectal cancer screening discussion and recommendation patterns. Cancer Epidemiol Biomarkers Prev. 2011;20(3):509-21.

39. Zuber AG, Lansdrop-Vogelaar I, Knudsen AB, Wilschut J, van Ballegooijen M, Kuntz DM. Evaluating test strategies for colorectal cancer screening: a decision analysis for the U.S. Preventive Services Task Force. Ann Intern Med. 2008; 149:659-669.

40. Levin B, Lieberman DA, McFarland B, et al. Screening and surveillance for the early detection of colorectal cancer and adenomatous polyps, 2008: a joint guideline from the American Cancer Society, the US Multi-Society Task Force on Colorectal Cancer, and the American College of Radiology. Gastroenterology. 2008;134(5):1570-1595.

41. DeBourcy AC, Lichtenberger S, Felton S, Butterfield KT, Ahnen DJ,Denberg TD. Community-based preferences for stool cards versus colonoscopy in colorectal cancer screening. J Gen Intern Med. 2008;23(2):169-74.

42. O'Malley AS, Beaton E, Yabroff KR, Abramson R, Mandelblatt J. Patient and provider barriers to colorectal cancer screening in the primary care safety-net. Prev Med. 2004;39(1):56-63.

43. Baker A, et al. Comparative effectiveness of a multifaceted intervention to improve adherence to annual colorectal cancer screening in community health centers. JAMA Intern Med. 2014;174(8):12351241. 